\title{
Coronary artery fistulas in children: Experience from a Southern African tertiary care centre complex
}

\author{
A.M. Cilliers", W. Hendson\#, F. Motara', P.E. Adams*, \\ G. Dumani" and H. Ntsinjana*
}

\section{"Division of Paediatric Cardiology, Chris Hani Baragwanath Academic Hospital, University of the Witwatersrand, Johannesburg, South Africa. \\ \#Department of Paediatrics, Rahima Moosa Maternal and Child Hospital, University of the Witwatersrand, Johannesburg, South Africa. \\ tDivision of Paediatric Cardiology, Charlotte Maxeke Johannesburg Academic Hospital, University of the Witwatersrand, Johannesburg, South Africa.}

\section{Address for correspondence:}

Professor Antoinette Cilliers

Paediatric Cardiology

Department of Paediatrics and Child Health

Chris Hani Baragwanath Academic Hospital

University of the Witwatersrand

PO Bertsham

2013

Johannesburg

South Africa

Email:

antoinette.cilliers@wits.ac.za

\section{INTRODUCTION}

Coronary artery fistulas (CAF) are rare vascular anomalies connecting coronary arteries with a low pressure cardiac chamber or vessel. The true incidence is not known because many are asymptomatic and only detected as an incidental murmur or when the patient undergoes an echocardiogram or cardiac catheterisation. Large CAF usually cause symptoms and may present with myocardial ischaemia or infarction, heart failure and arrhythmias. Surgical ligation and percutaneous embolisation have been used successfully as treatment modalities. A description of 6 cases presenting to an African tertiary care centre complex follows (Table I).

\section{CASE I}

A 12-year-old boy, presented with a 6 month history of dyspnoea associated with exercise. An innocent murmur had been diagnosed on clinical examination when he was younger but was not referred for further evaluation at the time. Clinical examination revealed a continuous murmur over the right parasternal area. The chest $\mathrm{X}$-ray showed mild cardiomegaly

\section{ABSTRACT}

Coronary artery fistulas (CAF) are unusual coronary artery connections with low pressure cardiac chambers or vessels. The majority are congenital, but can also be acquired. Complications include heart failure, myocardial infarction and arrhythmias. Symptomatic and large CAF require treatment and options include surgical ligation or percutaneous device embolisation of the fistula which has emerged as a less invasive and equally efficacious management modality. Careful interrogation of the CAF is required prior to occlusion in order not to compromise normal coronary artery vasculature that may arise from the fistula which can lead to myocardial ischaemia and infarction. Several reported cases highlight thrombus formation within large CAF after surgical ligation with propagation of the thrombus into coronary vessels arising proximally, resulting in myocardial compromise. We present a series of 6 children with CAF, 2 were treated by percutaneous embolisation (one developed a myocardial infarction post procedure) and $\mathbf{3}$ were treated surgically. SAHeart 2016; 1 3:20-26

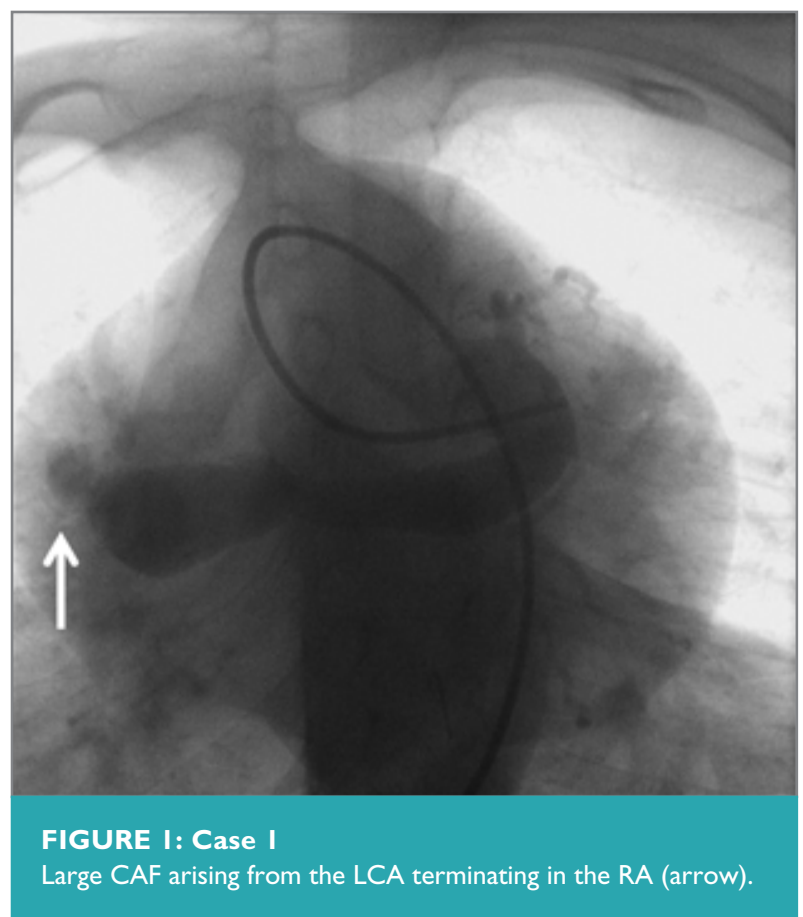


and the electrocardiogram (ECG) showed ST elevation in the inferior leads. An echocardiographic (ECHO) examination was diagnostic and showed a large left coronary artery (LCA) fistula terminating in the right atrium (RA). An angiogram confirmed the diagnosis of a large left CAF measuring $13 \mathrm{~mm}$ at its origin, tapering to $9 \mathrm{~mm}$, followed by a dilated area of $16 \mathrm{~mm}$ just before the termination point of $3 \mathrm{~mm}$ into the RA (Figure I). The left anterior descending (LAD) and circumflex arteries arose separately just distal to the origin of the CAF. The Qp/Qs ratio was calculated as 2.2:I and the mean pulmonary artery

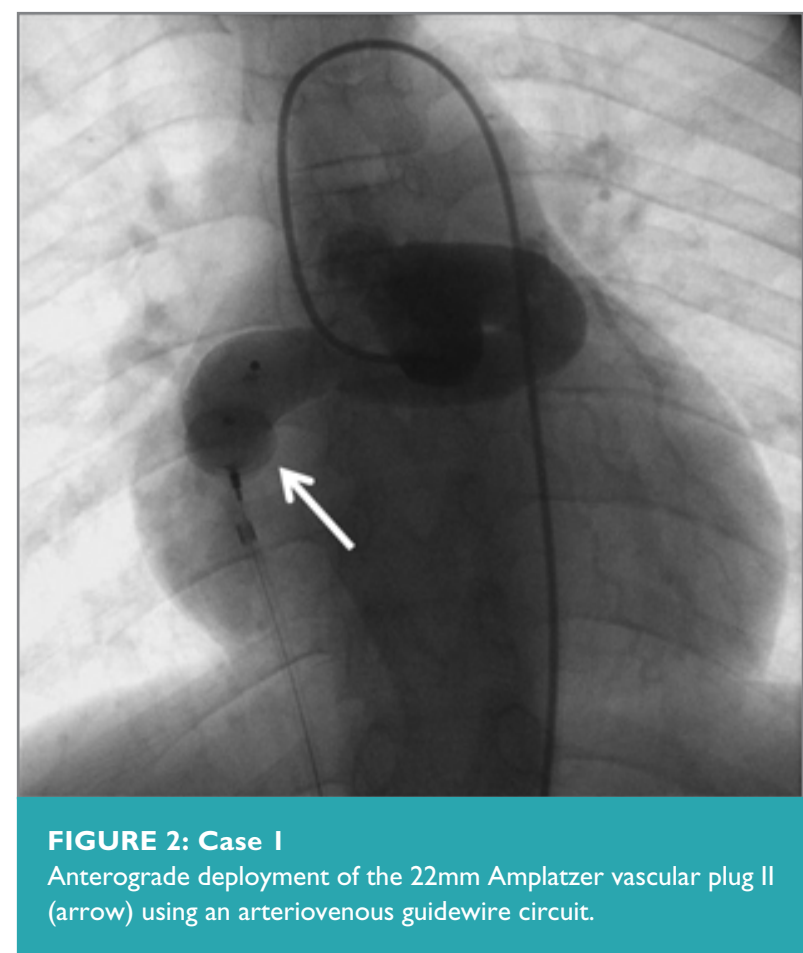

pressure was $22 \mathrm{mmHg}$. The CAF was occluded with a $22 \mathrm{~mm}$ Amplatzer Vascular plug II (St Jude Medical, USA) placed within the wider part of the fistula just proximal to its termination using an anterograde approach from the RA side over an arteriovenous guidewire loop (Figure 2). He was discharged without anticoagulation therapy. His symptoms improved, but presented 6 months later with severe crushing chest pain radiating to his back and both arms associated with shortness of breath. An ECHO showed new onset moderate mitral regurgitation and an urgent angiogram revealed an occluded

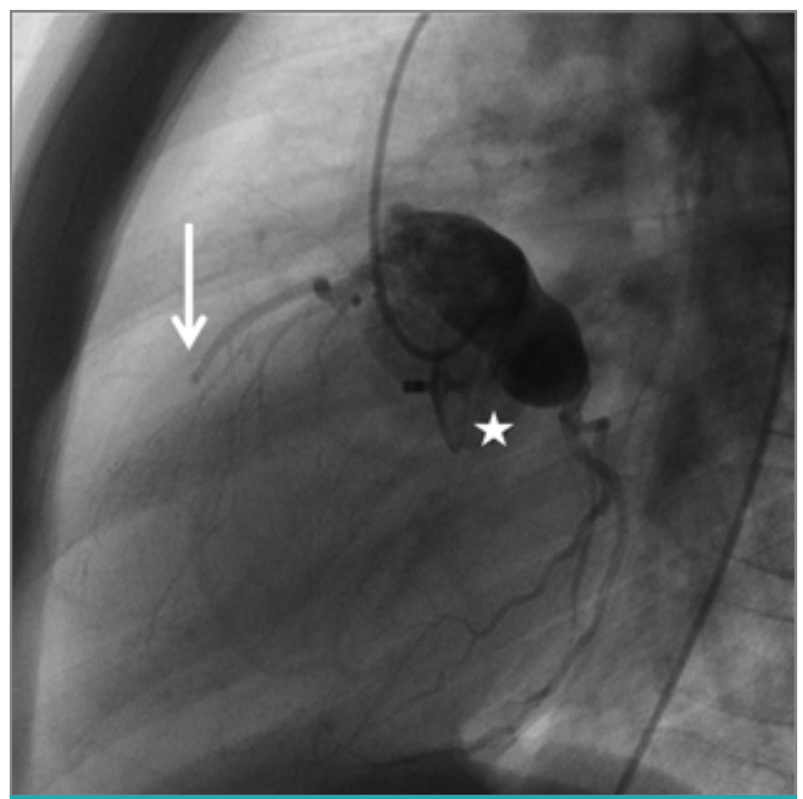

FIGURE 3: Case I

Angiogram in the lateral view 6 months later showing the device in-situ (star) with a dilated, aneurysmal LCA origin. The arrow shows the occluded LAD artery.

TABLE I: A description of 6 cases presenting to an African tertiary care centre complex.

\begin{tabular}{|c|c|c|c|c|c|c|c|}
\hline Case & Sex & Age & CAF & Termination & Presentation & Qp/Qs ratio & Associations \\
\hline । & M & 12 years & LCA & RA & Dyspnoea on exercise & $2.2: 1$ & - \\
\hline 2 & $\mathrm{~F}$ & 9 years & RCA & Coronary sinus & $\begin{array}{l}\text { Shortness of breath } \\
\text { and heart failure }\end{array}$ & $1.5: 1$ & - \\
\hline 3 & $M$ & 6 years & RCA & RV & Incidental murmur & $1.7: 1$ & Single coronary artery \\
\hline 4 & $M$ & 3 years & RCA & RV & Incidental murmur & $0.9: 1$ & - \\
\hline 5 & $\mathrm{~F}$ & 4 months & RCA & RA & $\begin{array}{c}\text { Cyanosis and } \\
\text { metabolic acidosis }\end{array}$ & Not calculated & Pulmonary atresia \\
\hline 6 & $\mathrm{~F}$ & 8 days & LCA & RV & $\begin{array}{l}\text { Heart failure and } \\
\text { metabolic acidosis }\end{array}$ & $\begin{array}{l}\text { Catheterisation } \\
\text { not done }\end{array}$ & $\begin{array}{l}\text { DSAS, aortic } \\
\text { regurgitation }\end{array}$ \\
\hline
\end{tabular}

$\mathrm{CAF}=$ coronary artery fistula, DSAS = discrete subaortic stenosis, $F=$ female, $L C A=$ left coronary artery, $M=$ male, $R A=$ right atrium, $R C A=$ right coronary artery, $R V=$ right ventricle. 


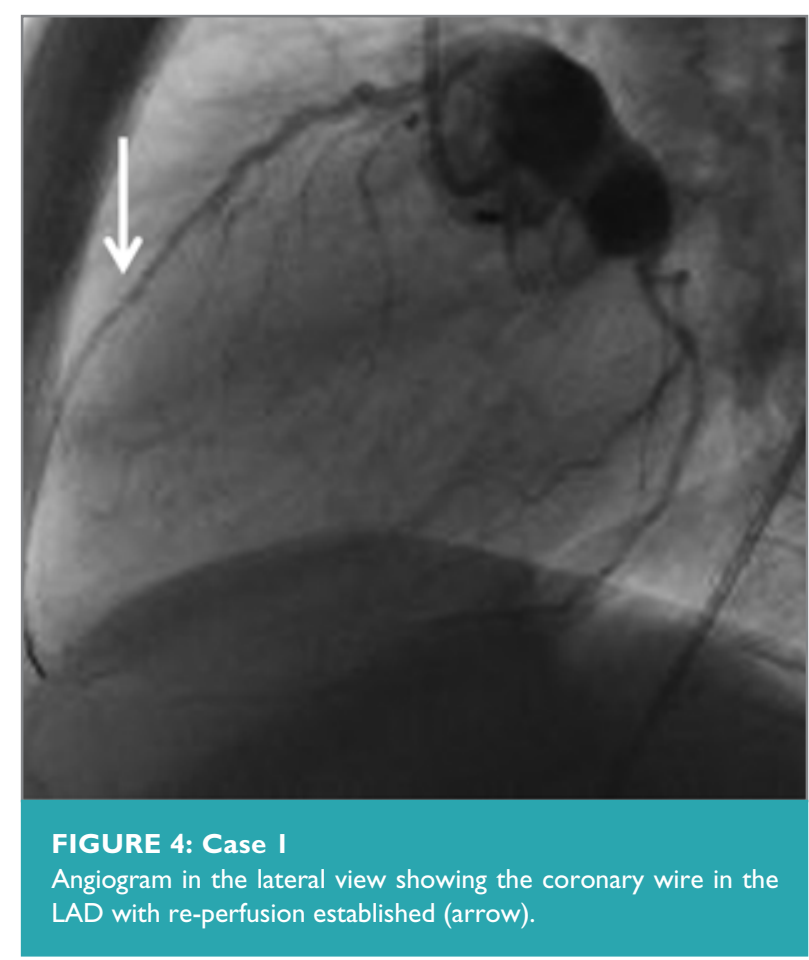

LAD artery and sluggish flow of contrast within the dilated origin of the CAF (Figure 3). The occluded LAD artery was cannulated with a coronary artery wire and sequential balloon angioplasty of the artery, along its length, was carried out using a coronary balloon. Re-cannulation of the LAD was established (Figure 4) and the patient was placed on warfarin and aspirin. A follow up $\mathrm{ECHO}$ showed mild mitral regurgitation and mildly reduced ventricular function with a shortening fraction of $24 \%$.

\section{CASE 2}

A 9-year-old female presented with a cough and shortness of breath on exertion. Clinical examination showed her to be wasted with features of right heart failure and a continuous murmur over the right infra-clavicular area. The chest $\mathrm{X}$-ray showed cardiomegaly and the ECG was normal. The ECHO demonstrated a dilated right coronary artery (RCA) measuring $9 \mathrm{~mm}$ consistent with a CAF terminating in a large coronary sinus, with the point of entry measuring $3 \mathrm{~mm}$. The diagnosis was confirmed at angiography (Figures 5 and 6). A small coronary vessel arose from the distal end of the fistula which appeared to perfuse the right ventricular (RV) apex (Figure 7). A Qp:Qs ratio of 1.5:I and a pulmonary vascular resistance (PVR) of 0.8 Wood units were calculated. Percutaneous occlusion of the CAF was considered but in view of the risk of occluding the small distal branch to the RV the patient was sent for surgical ligation. The post-surgery $\mathrm{ECHO}$ demonstrated good left ventricular function and the RCA artery origin remained dilated. The patient experienced severe menorrhagia with anaemia while on warfarin therapy post-surgery and is currently being maintained on low dose aspirin.

\section{CASE 3}

This 6-year-old boy presented with an incidental finding of a continuous murmur and cardiomegaly when he was admitted to hospital with gastroenteritis at the age of 4 years. The chest $\mathrm{X}$-ray confirmed cardiomegaly and the ECG was within normal

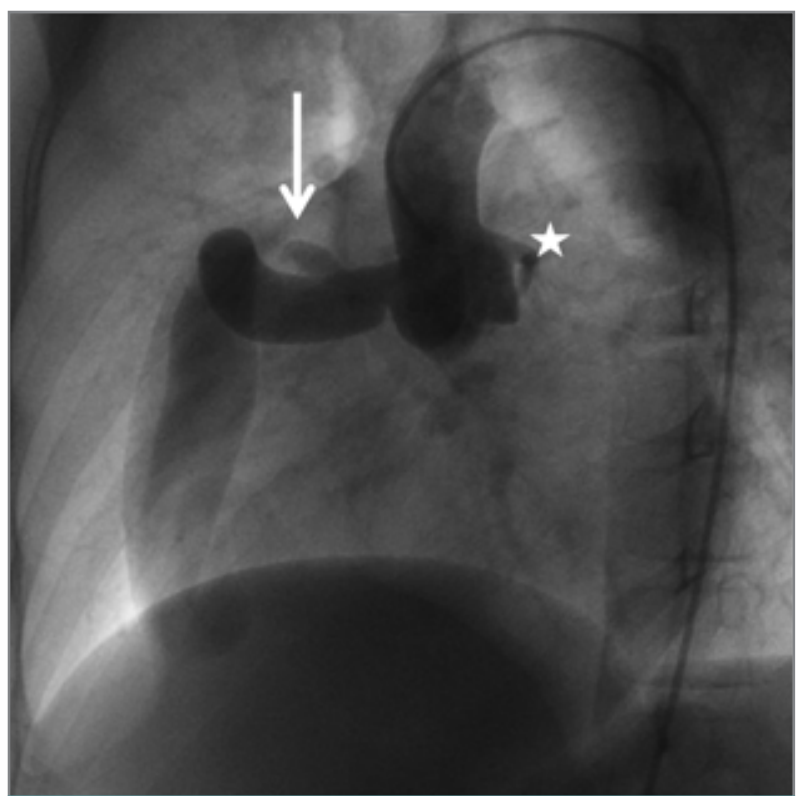

\section{FIGURE 5: Case 2}

An aortogram shows a large right CAF terminating in the coronary sinus. The RCA arises just beyond the origin of the CAF (arrow). A normal LCA originates from the left coronary sinus (star).

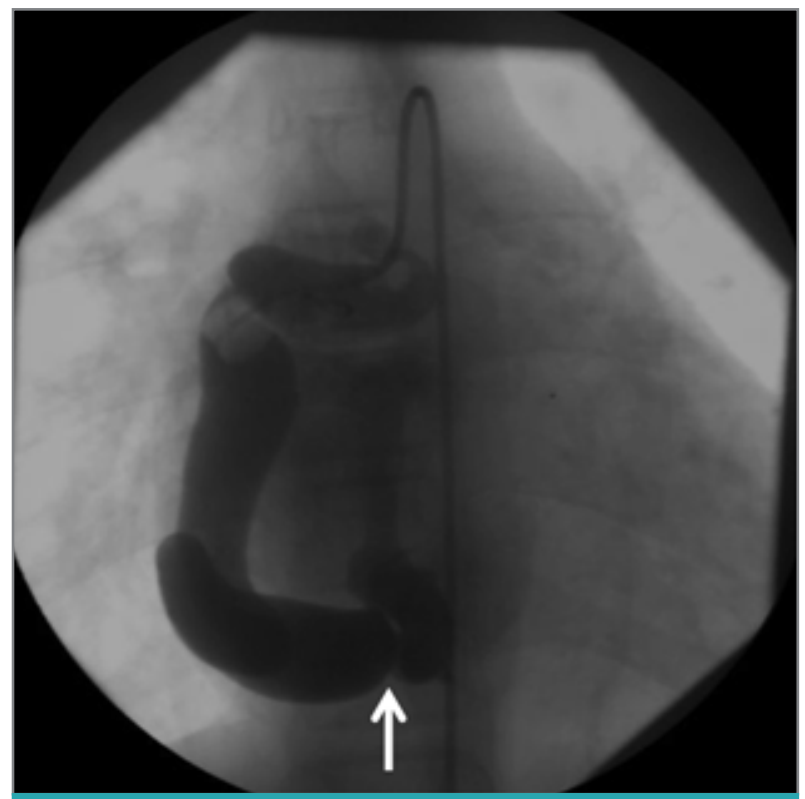

FIGURE 6: Case 2

The termination point of the CAF in the coronary sinus (arrow). 


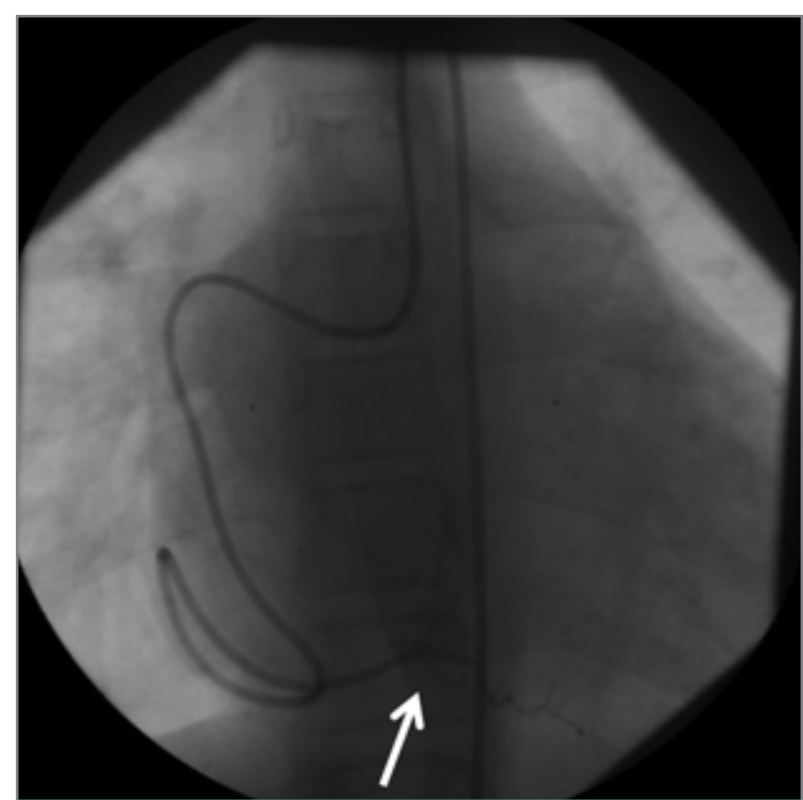

FIGURE 7: Case 2

A small coronary vessel arises from the distal end of the fistula (arrow) just before it enters the coronary sinus.

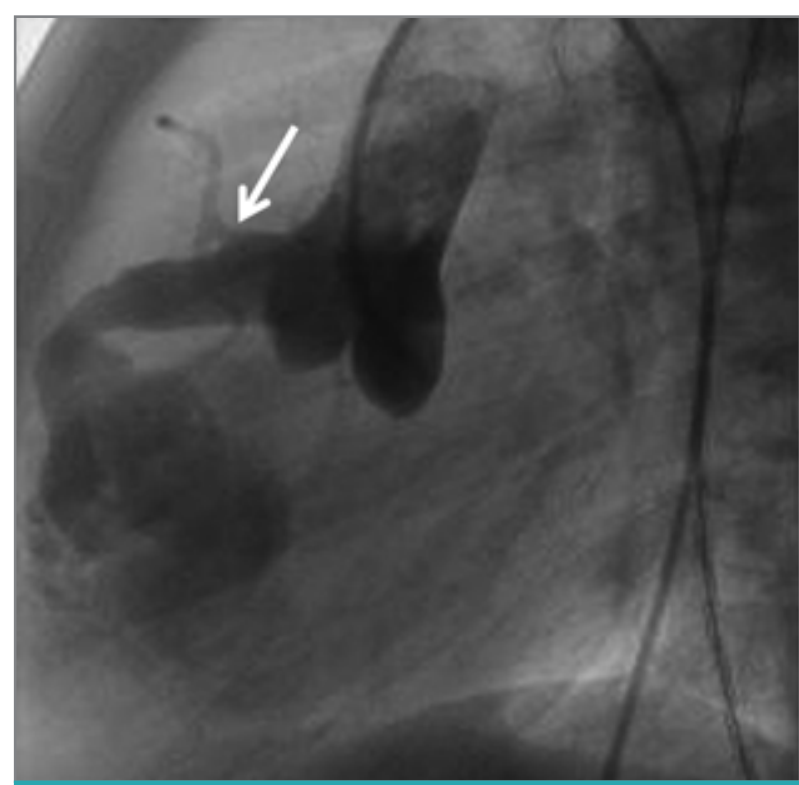

FIGURE 8: Case 3

An aortogram in the lateral view shows a large right CAF terminating in the RV. A common origin of the left and right coronary arteries is seen arising just distal to the origin of the fistula (arrow).

limits. An ECHO showed an aneurysmal RCA measuring I I mm in diameter communicating with the RV cavity. Angiography confirmed the presence of a CAF with a single common origin of both the right and left coronary arteries just distal to the origin of the fistula (Figure 8). A Qp:Qs of I.7:I and a normal
PVR were calculated. In view of the unusual anatomy and the single origin of the coronary arteries, surgical ligation of the terminal end of the fistula was undertaken. The RCA was found at surgery to give rise to the left main coronary artery which crossed the RV outflow tract. The CAF entered the RV in the middle of the atrioventricular groove. Aspirin therapy was prescribed post-surgery.

\section{CASE 4}

A 3-year-old boy was referred for assessment of a cardiac murmur which was found incidentally. Clinical examination revealed a continuous murmur over the lower left parasternal border. On direct questioning his mother disclosed reduced effort tolerance, he was not able to keep up with his peers during play. The chest X-ray and ECG were normal. The ECHO showed a large RCA measuring $4 \mathrm{~mm}$ in diameter. Angiography confirmed the diagnosis of a CAF terminating in the RV apex. Transient ST segment elevation was noted immediately after the selective angiogram of the RCA (Figure 9), the cause of

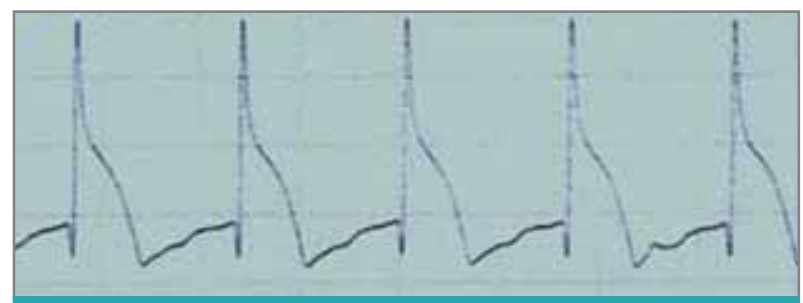

FIGURE 9: Case 4

Transient ischaemic changes noted on the electrocardiogram during selective angiography of the RCA.

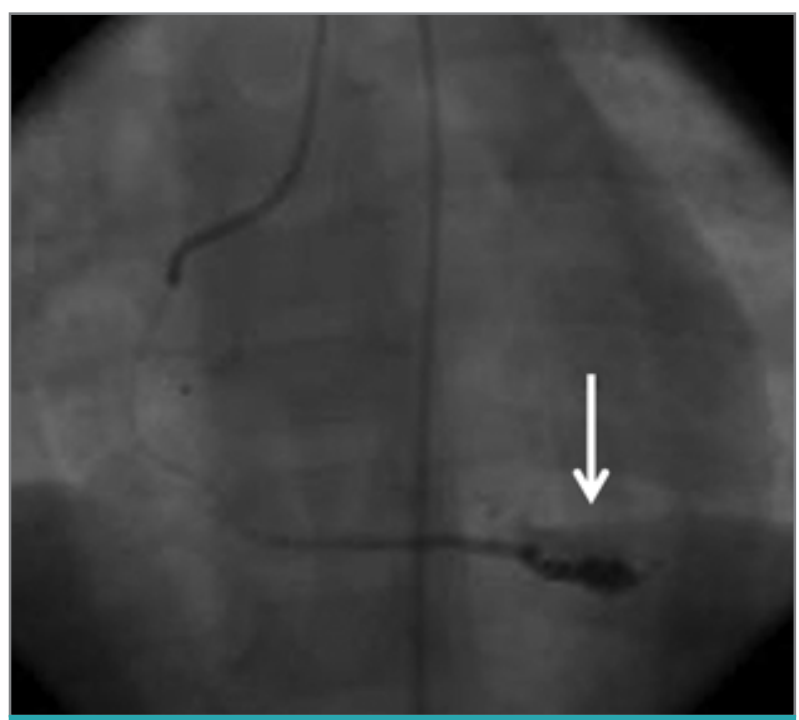

FIGURE I0: Case 4

End result after embolisation of the right CAF using both cyanoacrylate and two micro-coils at the termination point in the RV (arrow). 
which is speculative and may have been due to coronary artery spasm. The LCA, although normal in size, also connected with the fistula. A radiological glue, cyanoacrylate (Histoacryl, Melsungenm, Germany), was used to embolise the fistula through a Tracker microcatheter (Target Therapeutics, Boston Scientific Corporation, USA) at the terminal end of the RCA (Figure 10). A residual fistula noted at control angiography was occluded with two platinum micro-coils. The patient has remained well on follow up with no residual shunt.

\section{CASE 5}

This 4-month-old female infant presented with cyanosis and metabolic acidosis. An ECHO showed the patient to have pulmonary atresia, a ventricular septal defect, a right aortic arch with a left sided restrictive patent ductus arteriosus, a retroaortic innominate vein and a prominent RCA artery. Preoperative angiography showed the presence of a RCA to RA fistula (Figure 1 I). The patient had emergency surgery for a Blalock-Taussig shunt, but died afterwards from sepsis.

\section{CASE 6}

An 8-day-old female term infant with a birth weight of $3460 \mathrm{~g}$ presented with metabolic acidosis, respiratory failure and right heart failure requiring ventilator support. Cardiomegaly was present and a continuous murmur was audible at the cardiac apex. An enlarged pulsatile liver was palpable $7 \mathrm{~cm}$ below the right costal margin. The chest $\mathrm{X}$-ray showed a cardio-thoracic ratio of $73 \%$ and the ECG revealed ST depression and T-wave inversion in leads $\mathrm{V} 4$ and $\mathrm{V} 5$. The $\mathrm{ECHO}$ demonstrated a

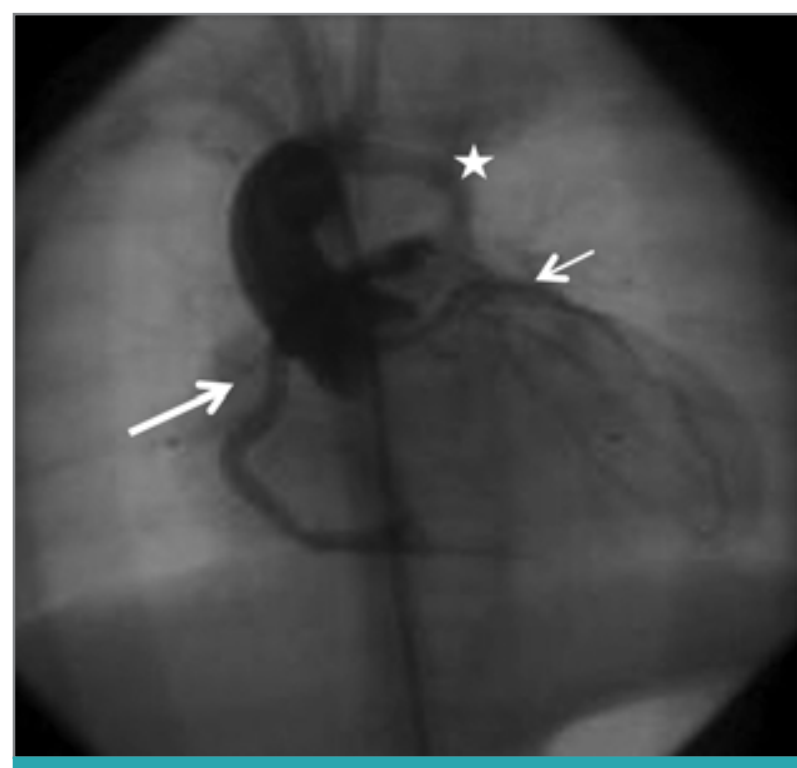

FIGURE I I: Case 5

An aortogram shows the large right CAF (large arrow) terminating in the RA. A normal LCA is present (small arrow). The aortic arch is right sided and a left sided patent ductus arteriosus (star) arises from the innominate artery.

dilated RV with a massive left CAF measuring IImm draining into the apex of the RV (Figure 12). A small $2 \mathrm{~mm}$ patent ductus arteriosus was present. There were also features of a discrete subaortic stenosis and moderate aortic regurgitation. Emer-
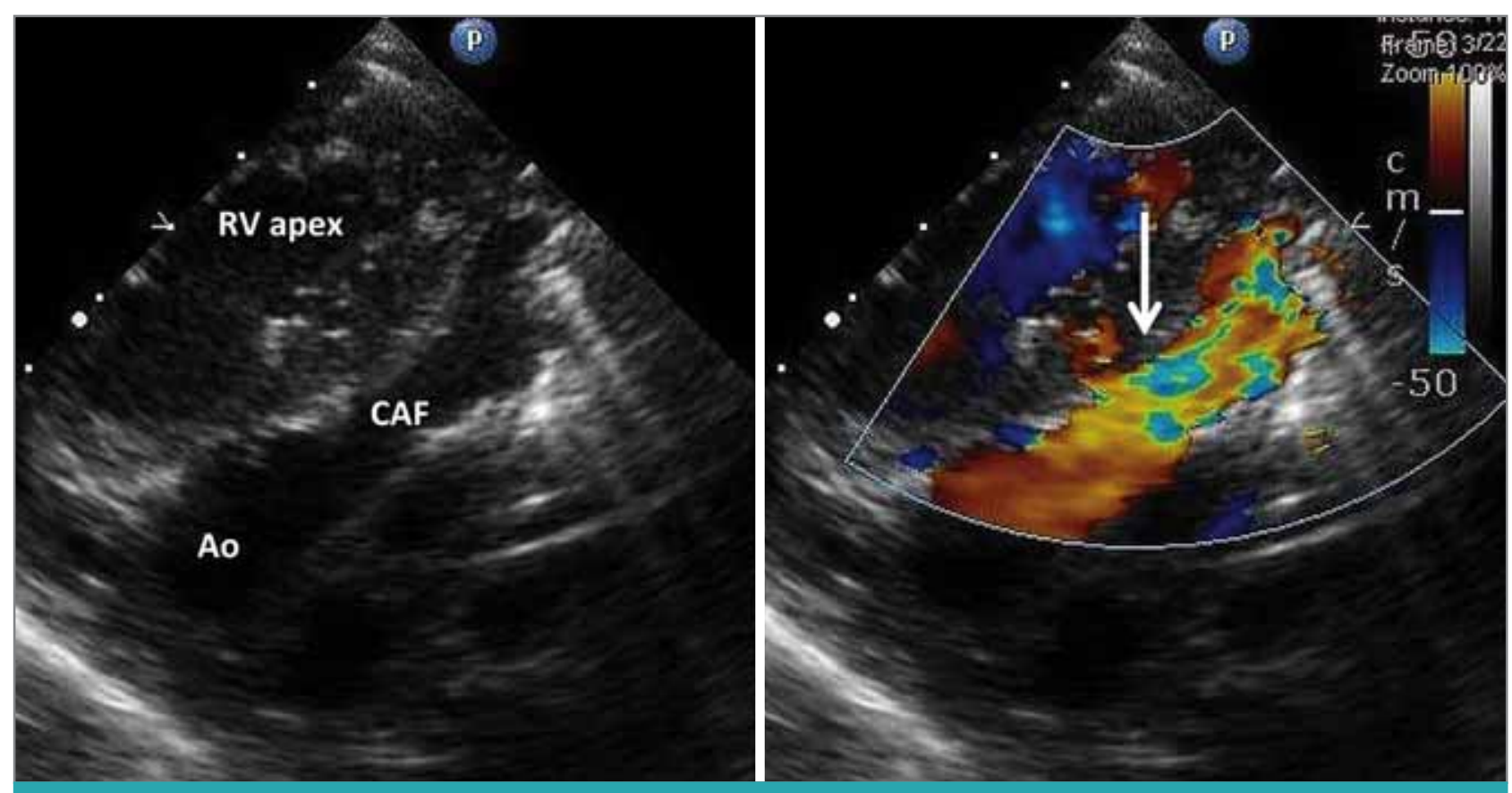

FIGURE I2: Case 6

An ECHO showing a large LCA to RV apex fistula. Ao, Aorta; CAF, coronary artery fistula; RV, right ventricle. The colour Doppler shows turbulent flow within the CAF (arrow). 
gency surgical ligation of the CAF was undertaken, but the patient died from intractable heart failure and sepsis after a protracted stay in the intensive care unit. The $\mathrm{ECHO}$ showed the CAF to be patent, but half the original size after surgery.

\section{DISCUSSION}

One case series of patients with $C A F^{(1)}$ several individual adult cases $^{(2-4)}$ and one child ${ }^{(5)}$ have been reported from the Southern African region over the last 3 - 4 decades. The case series from 2004 chronicled the percutaneous treatment of 4 patients ranging from 3 - 72 years. One of these cases (the 3-year-old) is included in this case series (Case 4) highlighting the use of cyanoacrylate glue as the primary transcatheter occlusive agent.(1) We present a second case series, summarised in Table I, comprising 6 children presenting with CAF from the neonatal period to 12 years of age. Their clinical presentation, anatomical features and treatment methods are similar to other cases reported in the literature. The potentially devastating complication of myocardial infarction (Case I) which can occur post-percutaneous embolisation or surgical ligation needs emphasis. Long term anticoagulation in patients with large CAF following occlusion should be considered and will be highlighted in the discussion that follows.

CAF are rare anomalies consisting of a direct precapillary communication between a coronary artery and a cardiac chamber, great vessel (usually the pulmonary artery) or other structures such as the coronary sinus and pulmonary veins, bypassing the myocardial capillary network. ${ }^{(6)}$ The fistulas originate from the RCA in about $52 \%$ of cases, LAD in $30 \%$ of cases and the circumflex coronary artery in about $18 \%$ of cases. ${ }^{(7)}$ They are usually congenital but can be acquired post chest trauma, cardiac intervention or surgery. A recent analysis of the angiograms of 16573 adult patients with symptomatic heart disease revealed a prevalence of $0.01 \% .{ }^{(8)}$ The true incidence is not known because 40 - 50\% of patients are asymptomatic and remain undiagnosed. ${ }^{(6)}$ About $20 \%$ of cases have been associated with additional congenital heart anomalies (Cases 3, 5 and 6). ${ }^{(9)}$

Indications for treatment are symptoms and complications which usually become apparent in older children and adults with large fistulas. Large fistulas cause symptoms due to coronary steal resulting in myocardial ischaemia. Heart failure due to a large left to right shunt especially in infancy ${ }^{(10)}$ (Case 6), dyspnoea on effort, arrhythmias, infective endocarditis and rupture have been described. ${ }^{(7,11-13)}$ The spontaneous closure of small CAF have been reported, therefore a conservative approach is appropriate in individuals with small fistulas. ${ }^{(14)}$ Asymptomatic patients with large fistulas need long term follow up because the disease has been shown to progress and patients can become symptomatic.(15)

Treatment methods depend on the anatomy of the fistula. Although surgery does have a place for selected cases (Cases 2 , 3 and 6) transcatheter occlusion is safe and effective, and has become the preferred method of treatment. The method of percutaneous occlusion has also evolved over time. Different embolisation materials have been used in the past and include detachable balloons, coils, cyanoacrylate, and in the last decade Amplatzer devices such as the vascular plug or duct occluders have been used successfully. ${ }^{(16,17)}$

The potential for device embolisation is present if the device chosen is too small. If a single device is used, the size chosen is roughly double the diameter of the fistula at its drainage point. (17) Complications related to the use of cyanoacrylate have been described with embolisation of the mixture into the pulmonary artery and lung in a patient with a CAF. The use of this type of embolisation method should be restricted to interventionalists experienced in its usage. (I)

Large fistulas, which are more than 3 times the expected size for the patient, ${ }^{(18)}$ with a relatively straight course may be treated with large occlusion devices such as the Amplatzer vascular plug using an arteriovenous guidewire circuit (Case I). ${ }^{(16)}$ Anterograde deployment of the device is possible through a large sheath. If the course is tortuous, coils can be used and deployed through an ordinary small French end hole catheter or a microcatheter in the case of micro-coils (Case 4). ${ }^{(16)}$

The final position of the occlusion device within the CAF is critical and should be placed distal to any coronary artery branches at the point of tapering of the fistula. Test occlusion for 10 minutes using a Berman or Swan-Ganz type of balloon catheter placed within the fistula to test for an ischaemic response prior to occlusion should be done.(5,16) Potential procedural complications include ischaemic changes up to 4 hours after occlusion, ${ }^{(15)}$ device embolisation and myocardial infarction.

Closure of the fistula results in relative stasis in the proximal giant coronary artery, and this is likely the aetiology of late thrombosis. ${ }^{(18)}$ Myocardial infarction due to thrombus formation in dilated CAF channels has been reported months (Case I) to years after surgical closure of CAF. A 26-year-old male patient reported in the literature with a large $L A D$ to right ventricle CAF, underwent surgical closure of a $10 \mathrm{~mm}$ orifice from within the dilated LAD. He presented 9 months later with severe sudden precordial pain. Angiography revealed a dilated CAF origin and complete occlusion of the LAD. ${ }^{(19)}$ A second report described a 14 -year-old girl who developed a myocardial infarction 10 years after surgical repair of a large LCA to RA fistula. She developed severe chest pain during a hockey game. Angiography demonstrated a thrombus into the LAD which extended from a large cul-de-sac of the original proximal CAF. Emergency treatment included the injection of Urokinase into the left main coronary artery (LMCA). Surgical reduction of the cul-de-sac origin of the LMCA followed. The patient had an uneventful recovery and was discharged on warfarin and aspirin. ${ }^{(20)}$ 
A report by Cheung, et al. ${ }^{(15)}$ suggests that coronary arteries may not return to normal following fistula occlusion. This large surgical series, post-operative angiograms performed at a mean follow-up period of 9 years, showed that the native coronary arteries remained dilated and tortuous, or more frequently had thrombus within the short proximal stump. The distal coronary arteries can remain normal in size but can also be threadlike or completely thrombosed, the circulation being maintained by retrograde filling from collaterals of the opposite coronary system. Residual and recurrent fistulas were also found in several patients. Hiraishi, et al. ${ }^{(21)}$ reported follow-up catheterisations and intravascular ultrasound studies in three patients, post-surgical repair of CAF. They showed areas of coronary artery stenosis and irregularities in the vessel wall due to intimal thickening at the site of regressed coronary artery aneurysms. The coronary artery findings were similar to those found after Kawasaki disease.

Routine follow-up catheterisation is recommended to assess for any residual aneurysmal areas or stenosis of coronary vessels. (22) Long term anticoagulation is advisable for patients with persistent coronary artery dilatation. ${ }^{(19,23,24)}$ Warfarin is recommended for the first 6 months to a year, followed by antiplatelet medication indefinitely. ${ }^{(24)}$

\section{CONCLUSION}

Although CAF are amenable to successful surgical or catheter treatment, there is a need for long term follow up. Large fistulas in particular require life-long anticoagulation if the parent fistula has an aneurysmal or dilated origin because of the risk of thrombus formation and propagation causing myocardial infarction that can occur months to years after treatment.

\section{Conflict of interest: none declared.}

\section{REFERENCES}

I. Duncan IC, Gebka M, Hellig F, et al. Percutaneous endovascular occlusion of symptomatic coronary arteriovenous fistulas with Cyanoacrylate. J Invasive Cardiol 2004; 16:469-74

2. Schamroth C. Coronary artery fistula. Images in Cardiology. J Am Coll Cardiol 2009;53:523.

3. Rose AG. Multiple coronary arterioventricular fistulae. Circulation 1978; 58:178-80

4. Bennett JM, Maree E. Successful embolisation of a coronary artery fistula. Int Journal of Cardiology 1989;23:405-6.

5. De Decker R, Comitis G, Border W, Degiovanni J. Coronary artery fistula occlusion. Image in Cardiology. SA Heart 20 I3; 10:472-74.

6. Said SA, El Gamal MI, Van der Werf T. Coronary arteriovenous fistulas: Collective review and management of 6 new cases - Changing etiology, presentation, and treatment strategy. Clin Cardiol 1997;20:748-52

7. McNamara JJ, Gross RE. Congenital coronary artery fistula. Surgery 1969:65:59-69.

8. Yuksel S, Yasar E, Nar G, et al. Prevalence and characteristics of CoronaryCameral communications in adult patients: Coronary angiographic analysis of 16573 patients. Med Princ Pract 2014;23:336-39.

9. Chih-Ta Lin, Tin-Kwang Lin. Current status of coronary artery fistula. J Intern Med Taiwan 2009;20:484-89.

10. Khan MD, Qureshi SA, Rosenthal E, et al. Neonatal transcatheter occlusion of a large coronary artery fistula with Amplatzer duct occluder. Catheter Cardiovasc Interv 2003;60:282-86

II. Wilde P, Watt I. Congenital coronary artery fistulae: Six new cases with a collective review. Clin Radiol 1980:31:301-11.

12. Alkhulaifi AM, Horner SM, Pugsley WB, et al. Coronary artery fistulas presenting with bacterial endocarditis. Ann Thorac Surg 1995;60:202-4.

13. Skimming JW, Wallis JT. Congenital coronary artery fistula suggesting a "steal phenomenon" in a neonate. Pediatr Cardiol 1993; 14:174-75.

14. Muthusamy R, Gupta G, Ahmed RA, et al. Fistula between a branch of left anterior descending coronary artery and pulmonary artery with spontaneous closure. Eur Heart J 1990; 1 1:954-56.

15. Cheung DL, Au WK, Cheung HH, et al. Coronary artery fistulas: Long-term results of surgical correction. Ann Thoracic Surg 2001;71:190-95.

16. Qureshi SA. Coronary arterial fistulas. Orphanet J Rare Dis 2006; 1:51.

17. Xian-Yang Zhu, Duan-Zhen Zhang, Xiu-Min Han, et al. Clin Cardiol 2009;32:506-12.

18. Latson LA. Coronary artery fistulas: How to manage them. Catheter Cardiovasc Interv 2007;70:1 10-16.

19. Hamada M, Kubo H, Matsuoka $\mathrm{H}$, et al. Myocardial infarction complicating surgical repair of left coronary-right ventricular fistula in an adult. Am J Cardiol 1986;57:372-74

20. Mesko ZG, Damus PS. Myocardial infarction in a 14 -year-old girl, 10 years after surgical correction of congenital coronary fistula. Pediatr Cardiol 1998; 19:366-68.

21. Hiraishi S, Misawa H, Horiguchi Y, et al. Effect of suture closure of coronary artery fistula of aneurysmal coronary artery and myocardial ischaemia. Am J Cardiol 1998;81:1263-67.

22. Fahey JT, Asnes J. Coronary recanalisation due to presumed thrombosis following surgical ligation of a large right coronary artery to right ventricle fistula. Congenit Heart Dis 2008:3:295-98.

23. Valente AM, Lock JE, Gauvreau K, et al. Predictors of long-term adverse outcomes in patients with congenital coronary artery fistulae. Circ Cardiovasc Interv 2010;3:134-39.

24. Gowda ST, Forbes TJ, Singh $\mathrm{H}$, et al. Remodelling and thrombosis following closure of coronary artery fistula with review of management: Large distal coronary artery fistula - to close or not to close? Catheter Cardiovasc Interv 2013;82:132-42 